\title{
Asymmetric Velocity Distributions from Halo Density Profiles in the Eddington Approach
}

\author{
J. D. Vergados ${ }^{1,2}$ \\ ${ }^{1}$ TEI of Western Macedonia, 50100 Kozani, Greece \\ ${ }^{2}$ University of Ioannina, 45110 Ioannina, Greece \\ Correspondence should be addressed to J. D. Vergados; vergados@uoi.gr
}

Received 9 July 2014; Accepted 11 September 2014

Academic Editor: Theocharis Kosmas

Copyright (c) 2015 J. D. Vergados. This is an open access article distributed under the Creative Commons Attribution License, which permits unrestricted use, distribution, and reproduction in any medium, provided the original work is properly cited. The publication of this article was funded by $\mathrm{SCOAP}^{3}$.

\begin{abstract}
We show how to obtain the energy distribution $f(E)$ in our vicinity starting from WIMP density profiles in a self-consistent way by employing the Eddington approach and adding reasonable angular momentum dependent terms in the expression of the energy. We then show how we can obtain the velocity dispersions and the asymmetry parameter $\beta$ in terms of the parameters describing the angular momentum dependence. From this expression, for $f(E)$, we proceed to construct an axially symmetric WIMP a velocity distribution, which, for a gravitationally bound system, automatically has a velocity upper bound and is characterized by the same asymmetriy $\beta$. This approach is tested and clarified by constructing analytic expressions in a simple model, with adequate structure. We then show how such velocity distributions can be used in determining the event rates, including modulation, in both the standard and the directional WIMP searches.
\end{abstract}

\section{Introduction}

The combined MAXIMA-1 [1-3], BOOMERANG [4, 5], DASI [6], and COBE/DMR cosmic microwave background (CMB) observations [7] imply that the Universe is flat [8] and that most of the matter in the Universe is Dark [9], that is, exotic. These results have been confirmed and improved by the recent WMAP [10] and Planck [11] data. Combining the data of these quite precise measurements one finds the following:

$$
\begin{gathered}
\Omega_{b}=0.0456 \pm 0.0015, \quad \Omega_{\mathrm{CDM}}=0.228 \pm 0.013, \\
\Omega_{\Lambda}=0.726 \pm 0.015
\end{gathered}
$$

(the more recent Planck data yield a slightly different combination $\Omega_{\mathrm{CDM}}=0.274 \pm 0.020, \Omega_{\Lambda}=0.686 \pm 0.020$ ). It is worth mentioning that both the WMAP and the Plank observations yield essentially the same value of $\Omega_{m} h^{2}$, but they differ in the value of $h$; namely, $h=0.704 \pm 0.013$ (WMAP) and $h=0.673 \pm 0.012$ (Planck). Since any "invisible" nonexotic component cannot possibly exceed $40 \%$ of the above $\Omega_{\mathrm{CDM}}$ [12], exotic (nonbaryonic) matter is required and there is room for cold dark matter candidates or WIMPs (weakly interacting massive particles).

Even though there exists firm indirect evidence for a halo of dark matter in galaxies from the observed rotational curves, see for example, the review [13], it is essential to directly detect such matter in order to unravel the nature of the constituents of dark matter. The possibility of direct dark matter detection, however, depends on the nature of the dark matter constituents (WIMPs). At present, there exists a plethora of such candidates: the LSP (lightest supersymmetric particle) [14-23], technibaryon [24, 25], mirror matter [26, 27], Kaluza-Klein models with universal extra dimensions $[28,29]$, and so forth.

Since the WIMP is expected to be very massive, $m_{\chi} \geq$ $30 \mathrm{GeV}$, and extremely nonrelativistic with average kinetic energy $T \approx 50 \mathrm{KeV}\left(m_{\chi} / 100 \mathrm{GeV}\right)$, it cannot excite the nucleus, except in some very exceptional cases. It can, thus, be directly detected mainly via the recoiling of a nucleus 
$(\mathrm{A}, \mathrm{Z})$ following WIMP-nucleus elastic scattering. The event rate for such a process can be computed following a number of steps [30]. In the present work, we will focus on one of the ingredients entering the computation of the event rates, namely, the WIMP density in our vicinity and its velocity distribution.

In the past, various velocity distributions have been considered. The one most commonly used is the isothermal Maxwell-Boltzmann (M-B) velocity distribution. Extensions of the M-B distribution were also considered; in particular, those that were axially symmetric with enhanced dispersion in the galactocentric direction [31-34]. In all such distributions, an upper cutoff $v_{\text {esc }}=2.84 v_{0}$ was introduced by hand, in the range obtained by Cochanek [35]. In a different approach, Tsallis type functions, derived from simulations of dark matter densities, were employed; see for example recent calculations [36] and references there in.

Nonisothermal models have also been considered, like the late infall of dark matter into the galaxy, that is, caustic rings [37-41], dark matter orbiting the Sun [42], and Sagittarius dark matter [43].

The more correct approach in our view is to consider the Eddington approach [44], which allows one to relate the dark matter density and the corresponding velocity distribution in a self-consistent way. Furthermore, this approach has the advantage that the upper velocity cut off is not imposed by hand, but it comes in naturally. It has, thus, been used by Merritt [45] and applied to dark matter by Ullio and Kamionkowski [13] and by us [46, 47].

It is the purpose of the present paper to extend the previous work and obtain a dark matter velocity distribution, which need not be spherically symmetric, even though it may originate from density profiles that are spherically symmetric. To this end, we have considered a one-parameter family of self-consistent star clusters that are spherically symmetric but anisotropic in velocity space. These were computed modifying the distribution (DF) by including suitable angular momentum factors along the lines suggested by Wojtak et al. [48] and more recently by Fornasa and Green [49]. Also a one-parameter family of self-consistent star clusters that are spherically symmetric was shown to be anisotropic in velocity space [50] (see also [51]). The last model was constructed first in the Newtonian limit and then, after the first, postNewtonian corrections were computed. Anisotropic velocity distributions obtained by adopting an ansatz for the dark matter phase space distribution. This allows one to construct self-consistent halo models, which feature a degree of anisotropy as a function of the radius such as suggested by the simulations [52]. Furthermore this has been applied [53] in the case of the NFW halo profile to obtain the asymmetry parameter.

To clarify some of the issues involved in these approaches, we will concentrate on some cases amenable to analytic solutions like the celebrated Plummer solution [54]. We will show how this method can be used to obtain, in a selfconsistent fashion, asymmetric velocity distributions with asymmetry parameter $\beta$. For detailed applications to dark matter searches realistic velocity distributions are necessary, but we leave this case to be discussed in a future publication.
We believe that even the prelude of such searches, as discussed here, falls within the novel subject of The Frontiers of Intensities and Very High Sensitivities.

\section{The Dark Matter Distribution in the Context of the Eddington Approach}

One assumes that the system is in steady state. This may not be exactly true, since simulated halos contain substructures corresponding to streams [55] and, more recently, to noncompletely phase-mixed DM, dubbed "debris flow" [56]. This, however, may be a reasonable assumption at the solar radius. Thus, in this approach, one starts with a phase space dark matter distribution function $f_{p s}(E, L)$, which is a function of the energy $E$ and the angular momentum $L$ with the goal of obtaining the dark matter velocity distribution $f_{r_{s}}(\boldsymbol{v})$. The function $f_{p s}(E, L)$ is factorized into a function $f(E)$, which depends on the energy only, and a function of the angular momentum $F_{L}(L)$. This factorization has been tested qualitatively by Wojtak et al. [48] and it has subsequently been discussed and used by [49]. Furthermore, these authors used the ansatz,

$$
F_{L}(L)=\left(1+\frac{L^{2}}{2 L_{0}^{2}}\right)^{-\beta_{\infty}+\beta_{0}} L^{-2 \beta_{0}},
$$

in terms of three new parameters. $L_{0}$ is an angular momentum parameter. This ansatz showed that the thus obtained self-consistent solutions match the radial dependence of the anisotropy parameter $\beta(r)$ (see below). The parameter $\beta_{0}$ affects the anisotropy in the cental region of the halo density, while $\beta_{\infty}$ has an effect at large distances [48]. To see this, we consider the limits previously considered; that is,

$$
F_{L}(L)= \begin{cases}\left(\frac{L}{L_{0}}\right)^{-2 \beta_{0}} & L \ll L_{0} \\ \left(\frac{L}{L_{0}}\right)^{-2 \beta_{\infty}} & L \gg L_{0},\end{cases}
$$

where $\beta_{0}$ is the the central anisotropy of the system. This means that, if this parameter is zero, there is no asymmetry in this region. In fact, it can be shown that $\beta_{0} \leq \gamma / 2$, where $\propto r^{-\gamma}$, is the halo density at the center. In the popular halo density profile [57], $\beta_{0} \leq 1 / 2$. In our analytically soluble model, we can better see the effect of these parameters on the asymmetry parameter.

2.1. The Distribution Is a Function of the Total Energy Only. The introduction of the matter distribution can be given [47] as follows:

$$
d M=2 \pi f\left(\Phi(\mathbf{r}), v_{r}, v_{t}\right) d x d y d z v_{t} d v_{t} d v_{r},
$$

where the function $f$ depends on $\mathbf{r}$ through the potential $\Phi(\mathbf{r})$ and the tangential and radial velocities $v_{t}$ and $v_{r}$. We will limit ourselves in spherically symmetric systems. Then, the density of matter $\rho(|r|)$ satisfies the following equation:

$$
d \rho=2 \pi f\left(\Phi(|\mathbf{r}|), v_{r}, v_{t}\right) v_{t} d v_{t} d v_{r}
$$


The energy is given by $E=\Phi(r)+v^{2} / 2$. Then,

$$
\begin{aligned}
\rho(r) & =4 \pi \int f\left(\Phi(r)+\frac{v^{2}}{2}\right) v^{2} d v \\
& =4 \pi \int_{\Phi}^{0} f(E) \sqrt{2(E-\Phi)} d E .
\end{aligned}
$$

This is an integral equation of the Abel type. It can be inverted to yield

$$
f(E)=\frac{\sqrt{2}}{4 \pi^{2}} \frac{d}{d E} \int_{E}^{0} \frac{d \Phi}{\sqrt{\Phi-E}} \frac{d \rho}{d \Phi} .
$$

The above equation can be rewritten as

$$
f(E)=\frac{1}{2 \sqrt{2} \pi^{2}}\left[\int_{E}^{0} \frac{d \Phi}{\sqrt{\Phi-E}} \frac{d^{2} \rho}{d \Phi^{2}}-\left.\frac{1}{\sqrt{-E}} \frac{d \rho}{d \Phi}\right|_{\Phi=0}\right]
$$

The potential $\Phi(r)$ for a given density $\rho(r)$ is obtained by solving Poisson's equation. In order to proceed further, it is necessary to know the density as a function of the potential, treating, for example, $r$ as a parameter. Only in few cases, this can be done analytically.

Once the function $f(E)$ is known, we can obtain the needed velocity distribution $f_{r_{s}}(v)$ in our vicinity $\left(r=r_{s}\right)$ by writing

$$
f_{r_{s}}\left(v^{\prime}\right)=\mathcal{N} f\left(\left.\Phi(r)\right|_{r=r_{s}}+\frac{v^{\prime 2}}{2}\right)
$$

where $\mathcal{N}$ is a normalization factor.

2.2. Angular Momentum Dependent Terms. As we have already mentioned in the phase distribution function, one introduces additional angular dependent terms. The presence of such terms can introduce asymmetries in the velocity dispersions.

In such an approach [48], we get

$$
\rho(\mathbf{r})=\iiint f(E)\left(1+\frac{L^{2}}{2 L_{0}^{2}}\right)^{-\beta_{\infty}+\beta_{0}} L^{-2 \beta_{0}} d^{3} \boldsymbol{v}
$$

Introducing the new parameters $L$ and $E$ in terms of $v_{t}$ and $v_{r}$ via

$$
\begin{aligned}
v_{t}=\frac{L}{r}, & v_{r}=\sqrt{2(E-\Phi)-\frac{L^{2}}{r^{2}}} \\
\text { or } \quad v_{t}=\frac{L_{0}}{r} \sqrt{2 \lambda}, \quad v_{r} & =\sqrt{2} \frac{L_{0}}{r} \sqrt{x-\lambda}, \quad \lambda=\frac{L^{2}}{2 L_{0}^{2}},
\end{aligned}
$$

we can perform the integration in cylindrical coordinates and get

$$
\rho(\mathbf{r})=2^{1 / 2-\beta_{0}} L_{0}^{1-2 \beta_{0}} \frac{\pi}{r} \int_{\Phi}^{0} f(E) d E \int_{0}^{x} \frac{\lambda^{-\beta_{0}}(\lambda+1)^{-\beta_{\infty}+\beta_{0}}}{\sqrt{x-\lambda}} d \lambda .
$$

In the above expressions, $x=\left(r^{2} / L_{0}^{2}\right)(\Phi-E)$.
Before proceeding further, we prefer to write the above formula in terms of dimensionless variables $\Phi=\Phi_{0} \xi, \rho=$ $\rho_{0} \eta, E=\Phi_{0} \epsilon$, and $f(E)=\rho_{0} \Phi_{0}^{-3 / 2} \tilde{f}(\epsilon)$. Thus, the last equation becomes

$$
\eta=2^{1 / 2-\beta_{0}} L_{0}^{-2 \beta_{0}} \frac{1}{\sqrt{a}} \pi \int_{\xi}^{0} \tilde{f}(\epsilon) d \epsilon \int_{0}^{x} \frac{\lambda^{-\beta_{0}}(\lambda+1)^{-\beta_{\infty}+\beta_{0}}}{\sqrt{x-\lambda}} d \lambda
$$

with $a=r^{2} \Phi_{0}^{2} / L_{0}^{2}$ and $x=a(\xi-\epsilon)$.

The second integral can be done analytically to yield

$$
\frac{\sqrt{\pi} x^{1 / 2-\beta_{0}} \Gamma\left(1-\beta_{0}\right)}{\Gamma\left(3 / 2-\beta_{0}\right)}{ }_{2} F_{1}\left(1-\beta_{0},-\beta_{0}+\beta_{\infty}, 3 / 2-\beta_{0},-x\right),
$$

with ${ }_{2} F_{1}$ the usual hypergeometric function. Then, (12) becomes

$$
\begin{aligned}
\eta= & 2^{1 / 2-\beta_{0}} L_{0}^{-2 \beta_{0}} \frac{1}{\sqrt{a}} \pi \frac{\sqrt{\pi} \Gamma\left(1-\beta_{0}\right)}{\Gamma\left(3 / 2-\beta_{0}\right)} \int_{\xi}^{0} \tilde{f}(\epsilon) d \epsilon x^{1 / 2-\beta_{0}} \\
& \times{ }_{2} F_{1}\left(1-\beta_{0},-\beta_{0}+\beta_{\infty}, 3 / 2-\beta_{0},-x\right) .
\end{aligned}
$$

In the limit in which $\beta_{0}->0, L_{0}->\infty$, the last expression is reduced to (6).

Equation (10) allows the calculation of moments of the velocity. In particular, following the procedure of [48], one finds

$$
\begin{aligned}
\prec & v_{t}^{2} \succ \\
= & 2\left(\frac{L_{0}}{r}\right)^{2}\left(2-\beta_{0}\right) \\
& \times \frac{\int_{\xi}^{0} \tilde{f}(\epsilon) d \epsilon x^{3 / 2-\beta_{0}}{ }_{2} F_{1}\left(2-\beta_{0},-\beta_{0}+\beta_{\infty}, 5 / 2-\beta_{0},-x\right)}{\int_{\xi}^{0} \tilde{f}(\epsilon) d \epsilon x^{1 / 2-\beta_{0}}{ }_{2} F_{1}\left(1-\beta_{0},-\beta_{0}+\beta_{\infty}, 3 / 2-\beta_{0},-x\right)} \\
\prec & v_{r}^{2} \succ \\
= & \left(\frac{L_{0}}{r}\right)^{2}\left(1-\beta_{0}\right) \\
& \times \frac{\int_{\xi}^{0} \tilde{f}(\epsilon) d \epsilon x^{3 / 2-\beta_{0}}{ }_{2} F_{1}\left(1-\beta_{0},-\beta_{0}+\beta_{\infty}, 5 / 2-\beta_{0},-x\right)}{\int_{\xi}^{0} \tilde{f}(\epsilon) d \epsilon x^{1 / 2-\beta_{0}}{ }_{2} F_{1}\left(1-\beta_{0},-\beta_{0}+\beta_{\infty}, 3 / 2-\beta_{0},-x\right)} .
\end{aligned}
$$

The extra factor of 2 in the case of the tangential velocity can be understood, since there exist two such components. The moments of the velocity are, of course, functions of the three parameters of the model. The model clearly can accommodate asymmetries in the velocity dispersion, even if the density is spherically symmetric.

Equation (12) can be inverted to yield the distribution function $\widetilde{f}(\epsilon)$, even though this is technically more complicated than in the standard Eddington approach without 
the angular momentum factors. Given the function $\tilde{f}(\epsilon)$, we define the quantities

$$
\begin{aligned}
\Lambda_{t}= & \left(2-\beta_{0}\right) \\
& \times \int_{\xi}^{0} \tilde{f}(\epsilon) d \epsilon_{2} F_{1}\left(2-\beta_{0},-\beta_{0}+\beta_{\infty}, 5 / 2-\beta_{0},-x\right), \\
\Lambda_{r}= & \left(1-\beta_{0}\right) \\
& \times \int_{\xi}^{0} \tilde{f}(\epsilon) d \epsilon_{2} F_{1}\left(1-\beta_{0},-\beta_{0}+\beta_{\infty}, 5 / 2-\beta_{0},-x\right) .
\end{aligned}
$$

Then, the asymmetry parameter $\beta$ defined by

$$
\beta=1-\frac{\prec v_{t}^{2} \succ}{2 \prec v_{r}^{2} \succ}, \quad \beta=1-\frac{\Lambda_{t}}{\Lambda_{r}} .
$$

The axially symmetric velocity distribution, with respect to the center of the galaxy, is, thus, obtained from $f(E)$ as described in the Appendix, for a number of cases, some of which, to the best of our knowledge, have not been obtained before in analytic form.

Clearly, for a given matter density profile, both the distribution function $\widetilde{f}(\epsilon)$ and the integrals $\Lambda_{t}$ and $\Lambda_{r}$ are functions of $r_{s} \beta_{0} \beta_{\infty}$ and $L_{0}$. So is the asymmetry parameter $\beta$. The above equations get simplified in the following cases.

(1) In the limit in which $\beta_{0}=0$ and $\beta_{\infty}=-1$,

$$
\begin{gathered}
\eta=4 \pi \int_{\xi}^{0} \tilde{f}(\epsilon) d \epsilon \sqrt{2(\epsilon-\xi)}\left(1+\frac{2}{3} a(\epsilon-\xi)\right), \\
a=\frac{r^{2} \Phi_{0}}{L_{0}^{2}} \\
\left\langle v_{t}^{2} \succ=\frac{2}{15} \frac{L_{0}^{2}}{r^{2}} \frac{\int_{\xi}^{0} \tilde{f}(\epsilon) d \epsilon(\epsilon-\xi)^{3 / 2}(5+4 a(\epsilon-\xi))}{\int_{\xi}^{0} \tilde{f}(\epsilon) d \epsilon \sqrt{\epsilon-\xi}(1+(2 / 3) a(\epsilon-\xi))}\right. \\
\left\langle v_{r}^{2} \succ=\frac{1}{15} \frac{L_{0}^{2}}{r^{2}} \frac{\int_{\xi}^{0} \tilde{f}(\epsilon) d \epsilon(\epsilon-\xi)^{3 / 2}(5+2 a(\epsilon-\xi))}{\int_{\xi}^{0} \tilde{f}(\epsilon) d \epsilon \sqrt{\epsilon-\xi}(1+(2 / 3) a(\epsilon-\xi))}\right. \\
\beta=1-\frac{\int_{\xi}^{0} \tilde{f}(\epsilon) d \epsilon(\epsilon-\xi)^{3 / 2}(5+4 a(\epsilon-\xi))}{\int_{\xi}^{0} \tilde{f}(\epsilon) d \epsilon(\epsilon-\xi)^{3 / 2}(5+2 a(\epsilon-\xi))} .
\end{gathered}
$$

(2) $\beta_{\infty}=1, \beta_{0}=0$.

In this case,

$$
\begin{aligned}
& \frac{1}{\sqrt{a}} x_{2}^{1 / 2-\beta_{0}}{ }_{2} F_{1}\left(1-\beta_{0},-\beta_{0}+\beta_{\infty}, 3 / 2-\beta_{0},-x\right) \\
& \longrightarrow \frac{1}{\sqrt{a}} \frac{\sinh ^{-1}(\sqrt{x})}{\sqrt{1+x}} .
\end{aligned}
$$

This function is very complicated to handle. Note, however, that, for sufficiently small values of $a$, one finds that the above expression for $x=a(\epsilon-\xi)$ is reduced to

$$
2 \sqrt{\epsilon-\xi}\left(1-\frac{2}{3} a(\epsilon-\xi)\right)
$$

We, thus, recover the previous formula with just a change of sign in $a$. The corresponding expressions for the velocity dispersions become

$$
\begin{gathered}
\Lambda_{t} \Longleftrightarrow 2\left(\sqrt{x}-\frac{\sinh ^{-1}(\sqrt{x})}{\sqrt{1+x}}\right), \\
\Lambda_{r} \Longleftrightarrow 4\left(-\sqrt{x}+\sqrt{1+x} \sinh ^{-1}(\sqrt{x})\right) .
\end{gathered}
$$

In the limit of small $a$, we again recover the previous expressions with $a \rightarrow-a$.

(3) The case of $L \gg L_{0}$ is as follows.

In this case, the integral equation

$$
\eta=\pi \sqrt{2 \pi} a^{-\beta_{\infty}} \frac{\Gamma\left(1-\beta_{\infty}\right)}{\Gamma\left(3 / 2-\beta_{\infty}\right)} \int_{\xi}^{0}(\epsilon-\xi)^{1 / 2-\beta_{\infty}} \tilde{f}(\epsilon) d \epsilon
$$

can be solved exactly (see Appendix) to yield

$$
\begin{aligned}
\tilde{f}(\epsilon)= & \frac{a^{\beta_{\infty}}}{\pi^{2} \sqrt{2 \pi}} \frac{\Gamma\left(3 / 2-\beta_{\infty}\right)}{\Gamma\left(1-\beta_{\infty}\right)} \frac{\sin \left(\pi\left(1 / 2-\beta_{\infty}\right)\right)}{\left(1 / 2-\beta_{\infty}\right)} \\
& \times \frac{d}{d \epsilon} \int_{\epsilon}^{0}(\xi-\epsilon)^{-1 / 2+\beta_{\infty}} \frac{d \eta(\xi)}{d \xi} d \xi
\end{aligned}
$$

provided that $\eta(0)=0$. In this case, however, we find that

$$
\beta=1-\frac{\Lambda_{t}}{\Lambda_{r}}=1-\frac{\Gamma\left(2-\beta_{\infty}\right)}{\Gamma\left(1-\beta_{\infty}\right)}=1-\beta_{\infty}, \quad \beta_{\infty}<1,
$$

regardless of the velocity distribution.

\section{Asymmetries in the Velocity Distribution}

Proceeding as above, we get the function $f_{\left(\beta_{\infty}, \beta_{0}, L_{0}\right)}(E)$. We, then, proceed to construct a velocity distribution, which is characterized by the same asymmetry in velocity dispersion along lines similar to those previously adopted [58], that is, by considering models of the Osipkov-Merritt type $[45,59$, 60]. Thus, the velocity distribution in our vicinity $\left(r=r_{s}\right)$ is written as

$$
f_{r_{s}}(\boldsymbol{v})=\mathcal{N}\left(1+\alpha_{s}\right) f_{0,0, \infty}\left(\Phi\left(r_{r} s\right)+\frac{v_{r}^{\prime 2}}{2}+\left(1+\alpha_{s}\right) \frac{v_{t}^{\prime 2}}{2}\right),
$$

where $v_{r}^{\prime}$ and $v_{t}^{\prime}$ are the radial, that is, outwards from the center of the galaxy, and the tangential components of the velocity, with respect to the center of the galaxy. The parameter $\alpha_{s}=\beta /(1-\beta)$ can be determined by calculating the moments of the velocity as above; that is, it is a function of the parameters $L_{0}, \beta_{0}$, and $\beta_{\infty}$. Since these parameters 
are usually treated as phenomenological parameters, we will treat $\beta$ phenomenologically. We note that this function is only axially symmetric and the normalization constant $\mathscr{N}$ is a normalization constant, the same as in the case of $\alpha_{s}=0$. The isotropic case follows as a special case in the limit $\alpha_{s} \rightarrow 0$.

The characteristic feature of this approach is that the velocity distribution automatically vanishes outside a given region specified by a cut-off velocity $v_{m}$, given by $v_{m}=$ $\sqrt{2\left|\Phi\left(r_{s}\right)\right|}$

\section{A Simple Test Density Profile}

Before proceeding further, we will examine a simple model, amenable to analytic solution, that is, the famous Plummer solution [54], and leave the case of realistic density profiles, like, for example, those often employed $[13,47,57]$, for a future publication. It is well known that a spherical density distribution [54] of the type

$$
\eta=\frac{\rho(x)}{\rho_{0}}=\frac{1}{\left(1+x^{2} / 3\right)^{5 / 2}}, \quad x=\frac{r}{a_{0}},
$$

which is sometimes used as an ordinary matter profile, leads to a potential of the form

$$
\xi=\frac{\Phi(x)}{\Phi_{0}}=-\frac{1}{\left(1+x^{2} / 3\right)^{1 / 2}}, \quad \Phi_{0}=4 \pi G_{N} a_{0}^{2} \rho_{0} .
$$

In the above expressions, $a_{0}$ is a scale length. More precisely, $a_{0}=3^{-1 / 6} R$ with $R$ being the radius of a sphere, containing the same mass, uniformly distributed. It is interesting to remark that the Plummer solution naturally arises in a model involving self-consistent star clusters studied in the Newtonian limit as well as after the first post-Newtonian corrections were computed [50].

From these, we obtain the desired relation:

$$
\begin{array}{r}
\eta(\xi)=-\xi^{5}, \quad \text { with } \eta^{\prime \prime}(\xi)=-20 \xi^{3}, \\
\left.\eta(\xi)\right|_{\xi=0}=0,\left.\quad \frac{d \eta}{d \xi}\right|_{\xi=0}=0 .
\end{array}
$$

Then, the solution to (19) is given by

$$
\begin{aligned}
\tilde{f}(x)= & \frac{16 e^{-a x}}{a^{9 / 2} \pi x} \\
& \times e^{a x}(\sqrt{a} \sqrt{x}(2 a x(2 a x(2 a x-5)+15)-15) \\
& +15 \sqrt{a x}) \\
& -15 a \sqrt{\pi} x \operatorname{erfi}(\sqrt{a x}), \quad x=-\epsilon .
\end{aligned}
$$

This leads to a velocity distribution:

$$
f_{\xi(x s)}(y)=\tilde{f}\left(\xi(x s)-\frac{y^{2}}{2}\right),
$$

where $\xi(x s)$ is the value of the potential in our vicinity. In our simple model $\xi(x s) \approx \sqrt{3} / 2$. We also used a larger value $\xi(x s)=10$.
(1) The choice $a>0$ is as follows.

The obtained velocity distribution which is properly normalized is exhibited in Figure 1. We notice that the dependence on $a$ is very mild.

We next compute the asymmetry parameter $\beta=1-$ $\Lambda_{t} / \Lambda_{r}$ as a function of the potential $\xi$ for various values of $a$. This is exhibited in Figure 2. The asymmetry is negative, opposite to what is commonly believed; see, for example, $[31-34,36,61]$; that is, it does not lead to enhanced dispersion in the galactocentric direction, regardless of the values of $\xi$. Thus, the positive values of $a$ are not acceptable; that is, the choice $\beta_{\infty}=-1, \beta_{0}=0$ is not physically acceptable.

(2) The choice $\beta_{\infty}=1, \beta_{0}=0$ is as follows.

In this case, we will explore the regime of negative absolutely small values of $a$, which is the physically interesting case. The velocity distribution obtained is exhibited in Figure 3, while the asymmetry parameter as a function of $\xi$, for the same values of $a$, is exhibited in Figure 4. Finally, the asymmetry parameter as a function of $a, a<0$, is presented in Figure 5 for various values of $\xi$.

\section{The Velocity Distribution in WIMP Searches}

The asymmetric velocity distribution in the galactic frame can be written as

$$
g\left(\beta, y^{\prime}\right)=\frac{1}{1-\beta} f_{0,0, \infty}\left(\Phi\left(r_{s}\right)+\frac{1}{2}\left(\frac{1}{1-\beta}\left(y^{\prime 2}-\beta y_{r}^{\prime 2}\right)\right)\right) .
$$

This function depends, of course, on the assumed density profile and is expressed in terms of two variables, the solar coordinate $r_{s}$ and the asymmetry parameter $\beta$. The latter depends on the parameters describing the angular momentum function $F_{L}(L)$. In order to get a feeling of what to expect in realistic calculations, we exhibit in Figure 6 the dependence on the asymmetry $\beta$ of the angular average of the distribution function obtained in our simple model. The values of $\beta$ employed were related to $a$ as above. The results shown here exhibit the same trends as those obtained by using, for example, Tsallis functions (see [36]).

Our next task is to transform the velocity distribution from the galactic to the local frame. The needed equation, see, for example, [62], is

$$
\begin{gathered}
\mathbf{y} \longrightarrow \mathbf{y}+\widehat{v}_{s}+\delta\left(\sin \alpha \hat{x}-\cos \alpha \cos \gamma \widehat{y}+\cos \alpha \sin \gamma \widehat{v}_{s}\right), \\
y=\frac{v}{v_{0}},
\end{gathered}
$$

with $\gamma \approx \pi / 6, \widehat{v}_{s}$ being a unit vector in the Sun's direction of motion, $\hat{x}$ a unit vector radially out of the galaxy in our position, and $\hat{y}=\widehat{v}_{s} \times \widehat{x}$. The last term in the first expression of (36) corresponds to the motion of the Earth around the Sun with $\delta$ being the ratio of the modulus of the Earth's velocity around the Sun divided by the Sun's velocity around the center of the Galaxy; that is, $v_{0} \approx 220 \mathrm{~km} / \mathrm{s}$ and $\delta \approx 0.135$. 


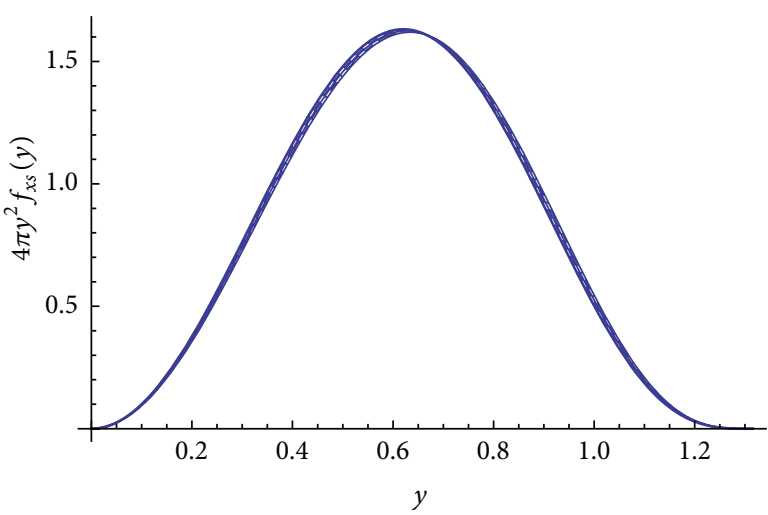

(a)

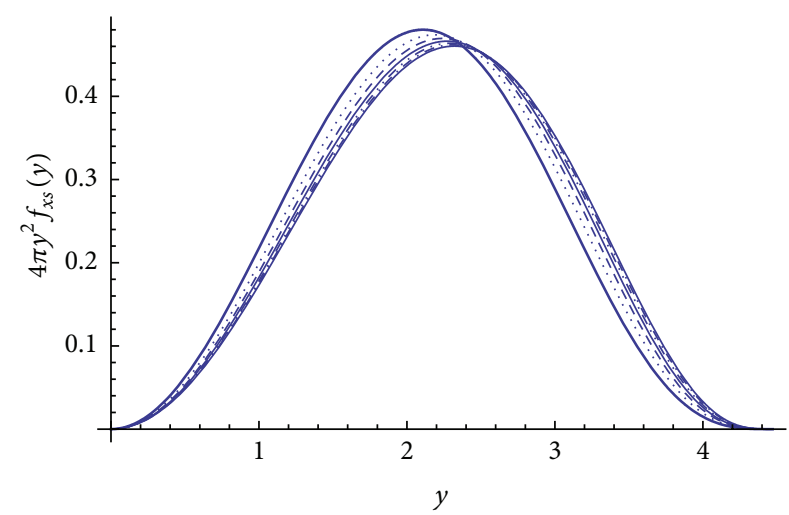

(b)

FIGURE 1: We show the properly normalized velocity distribution obtained in our simple model for various values of $a$ for the value $\xi(x s)=$ $\sqrt{3} / 2$ (a) and a larger, perhaps more realistic, value $\xi(x s)=10$ (b). The obtained velocity distribution depends mildly on $a$.

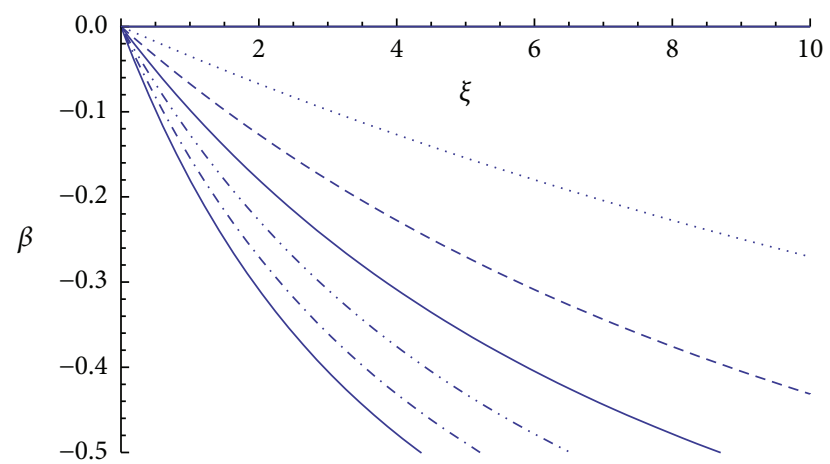

FIgURE 2: The asymmetry parameter $\beta=\Lambda_{t} / \Lambda_{r}$ as a function of $\xi$ for values of $a=0,0.25,0.50,0.75,1.0,1.25,1.50$ increasing downwards.

The above formula assumes that the motion of both the Sun around the Galaxy and the Earth around the Sun is uniformly circular. The exact orbits are, of course, more complicated $[63,64]$, but such deviations are not expected to significantly modify our results. In (36), $\alpha$ is the phase of the Earth $(\alpha=$ 0 around June $3 \mathrm{rd}$ ). (One could, of course, make the time dependence of the rates, due to the motion of the Earth, more explicit by writing $\alpha \approx(6 / 5) \pi(2(t / T)-1)$, where $t / T$ is the fraction of the year).

5.1. Standard Nondirectional Experiments. We have seen that, in the galactic frame, in the presence of asymmetry $\beta$, the relevant quantity is

$$
y_{x}^{\prime 2}+\frac{1}{1-\beta}\left(y_{y}^{\prime 2}+y_{z}^{\prime 2}\right)=\frac{1}{1-\beta}\left(y^{\prime 2}-\beta y_{x}^{\prime 2}\right)
$$

In the local frame, the components $y_{x}, y_{y}, y_{z}$ of the velocity vector $\mathbf{y}$ are, thus, given by

$$
\begin{gathered}
y_{r}=y_{x}=\frac{1}{s c}(y \cos \phi \sin \theta+\delta \sin \alpha), \\
y_{t}=\sqrt{y_{y}^{2}+y_{z}^{2}} \\
y_{y}=\frac{1}{s c}(y \sin \theta \sin \phi-\delta \cos \alpha \cos \gamma), \\
y_{z}=\frac{1}{s c}(y \cos \theta+\delta \cos \alpha \sin \gamma+1), \\
y=\frac{v}{v_{0}},
\end{gathered}
$$

where $s_{c}$ is a suitable scale factor to bring the WIMP velocity into units of the Sun's velocity, $y=v / v_{0}$; that is, $s c=$ $\sqrt{\left|\Phi_{0}\right|} / v_{0}$. One finds

$$
\begin{aligned}
\frac{1}{1-\beta}\left(y^{\prime 2}-\beta y_{x}^{\prime 2}\right) & \\
\longrightarrow Y^{2} & \\
=\frac{1}{s c^{2}} \frac{1}{1-\beta} & \left(-\beta(\delta \sin (\alpha)+y \cos (\phi) \sin (\theta))^{2}\right. \\
+ & (y \cos (\theta)+\delta \cos (\alpha) \sin (\gamma)+1)^{2} \\
+ & \left.(\delta \cos (\alpha) \cos (\gamma)-y \sin (\theta) \sin (\phi))^{2}\right) .
\end{aligned}
$$

Thus, the velocity distribution for the standard (nondirectional) case becomes

$$
g_{\text {nodir }}(Y)=\frac{1}{1-\beta} f_{0,0, \infty}\left(\Phi\left(r_{s}\right)+\frac{1}{2} Y^{2}\right) .
$$




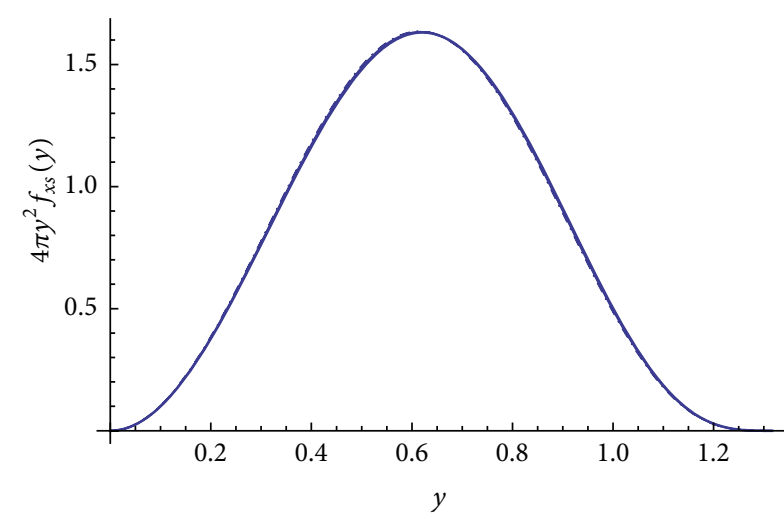

(a)

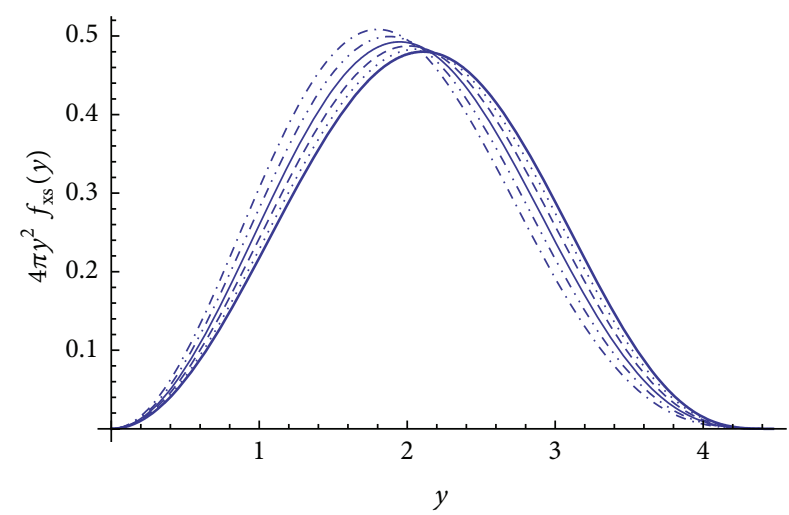

(b)

FIGURE 3: We show the properly normalized velocity distribution obtained in our simple model for negative values of $a$; that is, $a=$ $0,-0.1,-0.2,-0.3,-0.4,-0.5$ for the value $\xi(x s)=\sqrt{3} / 2$ (a) and a larger, perhaps more realistic, value $\xi(x s)=10$ (b). The obtained velocity distribution depends mildly on $a$ in (a) and it is noticeable in (b). In the plots, $a$ is increasing from left to right.

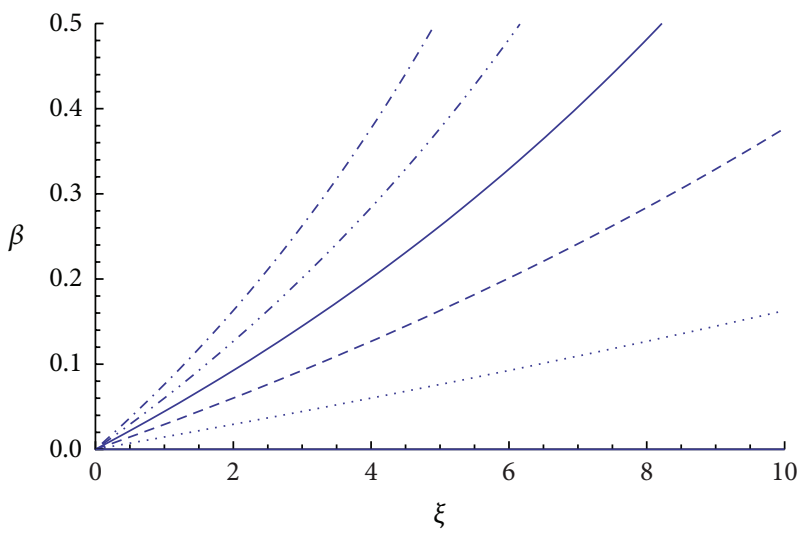

Figure 4: The asymmetry parameter $\beta=\Lambda_{t} / \Lambda_{r}$ as a function of $\xi$ for values of $a$ is the same as in Figure 3. In the plots, $a$ is increasing upwards.

5.2. Directional Experiments. In the Eddington theory the asymmetric velocity distribution is given by

$$
g_{\mathrm{dir}}(X)=\frac{1}{1-\beta} f_{0,0, \infty}\left(\Phi\left(r_{s}\right)+\frac{1}{2} X^{2}\right)
$$

where $f$ is the symmetric normalized velocity distribution with respect to the center of the galaxy, $\beta$ is the asymmetry parameter, and $X$ is given, [65], by

$X^{2}$

$$
\begin{aligned}
= & \frac{1}{(1-\beta) s_{c}^{2}} \\
& \times\left(\sqrt{3} \delta \cos \alpha \cos \Phi-2 \sqrt{1-\xi^{2}} \sin \phi+2 \delta \sin \alpha \sin \Phi\right)^{2}
\end{aligned}
$$

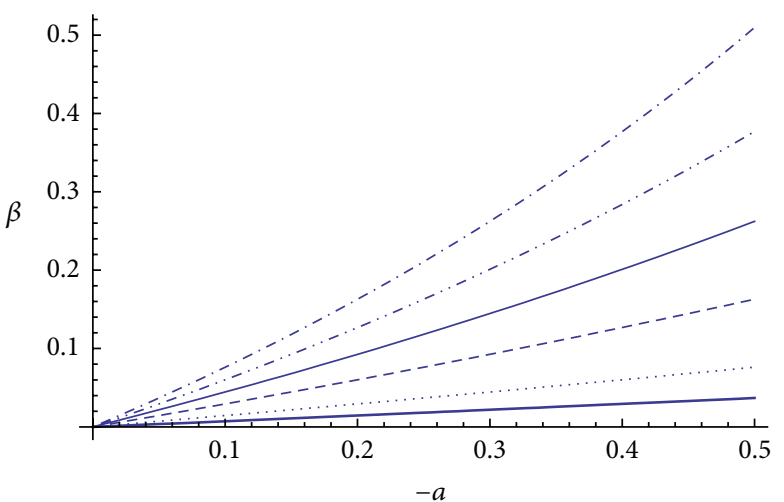

FIGURE 5: The asymmetry parameter $\beta=\Lambda_{t} / \Lambda_{r}$ as a function of $a$ for values of $\xi\left(r_{s}\right)=(1,2,4,6,8,10) / 2$. In the plots $\xi\left(r_{s}\right)$ is increasing upwards. Note that, on the $x$-axes, the opposite of $a$ is indicated.

$$
\begin{aligned}
& -\beta\left(2 \sqrt{1-\xi^{2}} \cos \phi-(\delta \cos \alpha+2) \sin \Theta\right. \\
& +\delta \cos \Theta(2 \cos \Phi \sin \alpha-\sqrt{3} \cos \alpha \sin \Phi))^{2} \\
& +(2 \xi y+(\delta \cos \alpha+2) \cos \Theta \\
& +\delta \sin \Theta(2 \cos \Phi \sin \alpha-\sqrt{3} \cos \alpha \sin \Phi))^{2} \\
& +\left(-2 \sqrt{1-\xi^{2}} \cos \phi+(\delta \cos \alpha+2) \sin \Theta\right. \\
& +\delta \cos \Theta(\sqrt{3} \cos \alpha \sin \Phi-2 \cos \Phi \sin \alpha))^{2} .
\end{aligned}
$$

The direction of the WIMP velocity is specified by $\xi=\cos \theta$ and $\phi$. The direction of observation is specified by the angles $\Theta$ and $\Phi$. 


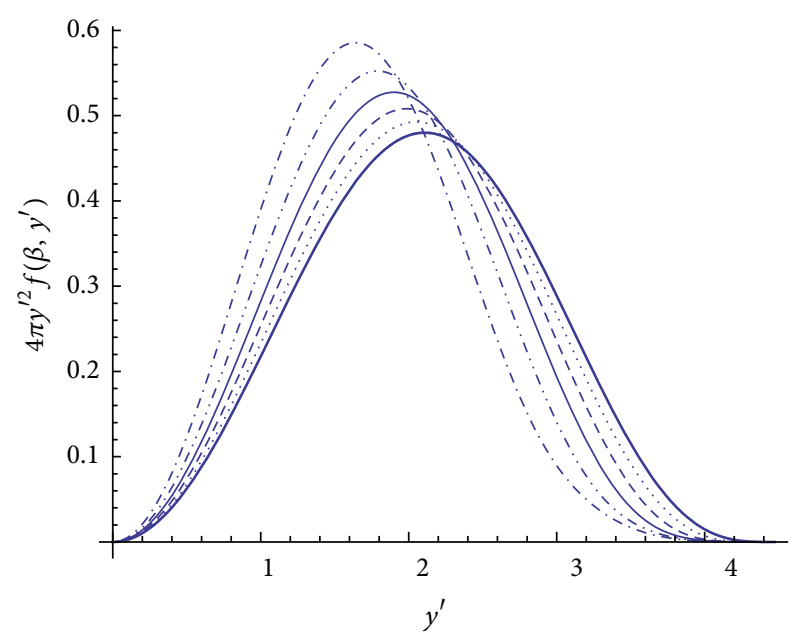

FIGURE 6: We show the angular average of the properly normalized velocity distribution for values of the asymmetry parameter $\beta=$ $(0.0 .1,0.2,0.3,0.4,0.5)$. In the plots, $\beta$ is increasing from right to left. The results depend on the value of the potential in our vicinity. Here, the value of $\xi(x s)=10$ was adopted.

\section{Discussion and Conclusions}

In the present work, we studied how one can construct the velocity distribution in the Eddington approach starting from dark matter density profiles. This is very important in the case of using this distribution for calculating the event rates expected in direct dark matter searches. First, because it allows a consistency between the velocity distribution employed and the WIMP density in our vicinity. Second, because the upper cut-off in the velocity distribution comes out of the model and is not put in by hand as is common practice. It is, therefore, interesting to generalize the Eddington approach in order to obtain asymmetric velocity distributions.

With this in mind, we have seen that, by modifying the phase space distribution function by suitable angular momentum functions $F_{L}(L)$, one can obtain asymmetric velocity distributions as well, with $\alpha v$ asymmetry parameter $\beta$, which is described in terms of the parameters specifying $F_{L}(L)$. We clarified some of the issues involved in this approach by considering a simple model, which can yield analytic solutions.

Results of realistic calculations for dark matter searches, employing the present technique and using realistic density profiles [13, 47,57], will appear elsewhere [66]. We do not expect the effects of the asymmetry on the standard nondirectional rates to be very different from those obtained in a more phenomenological treatment [36], that is, negligible in the case of time averaged events and small in the case of time dependent rates (modulation effect due to the motion of the Earth). We expect, however, the effects of asymmetry to be very important in the case of directional experiments, that is, experiments measuring not only the energy but also the direction of the recoiling nucleus. Even though velocity distributions without asymmetry [65] were employed, it has been found that there is a strong dependence of the event rates on the angle of observation relative to the direction of the velocity of the Sun, for both the time averaged and the modulated events.

\section{Appendix}

\section{Analytic Solutions of Some Integral Equations}

Consider an integral equation of the following form:

$$
\int_{0}^{x} f(y) K(x-y) d y=g(x) .
$$

Applying the Laplace transform on both sides, this is reduced to

$$
L(f) L(K(t))=L(g) \Longrightarrow L(f)=\frac{L(g)}{L(K(t))}
$$

The solution can be obtained if we can find a function $\widetilde{K}(t)$ such that $L(\widetilde{K})=1 / L(K)$. This, however, cannot be done analytically except in very few cases. Some cases of interest are as follows:

(1) $K(t)=(\Gamma(1-v) / \Gamma(3 / 2-v)) t^{1 / 2-\nu}$.

Then, one can show that

$L\left(\frac{x^{\nu-5 / 2}}{\Gamma(1-\nu) \Gamma(\nu-3 / 2)}\right)=\left(\frac{\Gamma(1-\nu)}{\Gamma(3 / 2-\nu)} t^{1 / 2-\nu}\right)^{-1}$

or

$$
L\left(\frac{t^{-2-\nu}}{\Gamma(-1-v) \Gamma(1+\nu)}\right)=\frac{1}{L\left(t^{\nu}\right)}
$$

Thus,

$$
\begin{aligned}
& g(x)=\int_{0}^{x} f(y)(x-y)^{\nu} d y \Longrightarrow \\
& f(x)=\frac{1}{\Gamma(-1-\nu) \Gamma(1+\nu)} \int_{0}^{x}(x-y)^{-2-v} g(y)
\end{aligned}
$$

or better still

$$
\frac{1}{\Gamma(1+\nu) \Gamma(1-\nu)} s L\left(t^{-\nu}\right) s=\frac{1}{L\left(t^{\nu}\right)}, \quad-1<\Re(\nu)<1,
$$

where

$$
s \Longleftrightarrow \frac{d}{d t} \Longleftrightarrow L\left(\frac{d u}{d t}\right)=s L(u), \quad u(0)=0 .
$$

Thus,

$$
\begin{aligned}
& g(x)=\int_{0}^{x} f(y)(x-y)^{\nu} d y \Longrightarrow \\
& f(x)=\frac{1}{\pi} \frac{\sin \pi \nu}{\nu} \frac{d}{d x} \int_{0}^{x}(x-y)^{-\nu} \frac{d g(y)}{d y}, \quad-1<\Re(\nu)<1 .
\end{aligned}
$$


(2)

$K(t)=\sqrt{t}\left(1+\frac{2}{3} a t\right) \Longrightarrow L(K)=\sqrt{\pi} \frac{1}{2 s^{5 / 2}}(a+s)$,

which is a special case of (19).

Then, we notice that

$$
\begin{array}{r}
\frac{L(g)}{L(K(t))}=s L\left(\frac{2 e^{-a t} \operatorname{erfi}(\sqrt{a} \sqrt{t})}{\sqrt{a} \sqrt{\pi}}\right) L\left(g^{\prime \prime}\right), \\
\text { if } g(0)=0, \quad g^{\prime}(0)=0,
\end{array}
$$

where erfi is the error function with imaginary part; that is,

$$
\operatorname{erfi}(x)=-i \operatorname{erf}(i x)
$$

Thus, the solution becomes

$$
f(x)=\frac{d}{d x} \int_{0}^{x}\left(\frac{2 e^{-a(x-y)} \operatorname{erfi}(\sqrt{a} \sqrt{(x-y)})}{\sqrt{a} \sqrt{\pi}}\right) g^{\prime \prime}(y) \text {. }
$$

Thus, the solution of (19) takes the following form:

$$
\tilde{f}(\epsilon)=\frac{1}{2 \pi \sqrt{2 \pi}} \frac{d}{d \epsilon} \int_{\epsilon}^{0}\left(\frac{e^{-a(\xi-\epsilon)} \operatorname{erfi}(\sqrt{a} \sqrt{(\xi-\epsilon)})}{\sqrt{a}}\right) \eta^{\prime \prime}(\xi) .
$$

This reduces to (8) in the limit of $a \rightarrow 0$.

\section{Conflict of Interests}

The author declares that there is no conflict of interests regarding the publication of this paper.

\section{Acknowledgments}

This research has been partially supported by the European Union Social Fund (ESF) and the Greek national funds through the program THALIS of the Hellenic Open University: Development and Applications of Novel Instrumentation and Experimental Methods in Astroparticle Physics.

\section{References}

[1] S. Hanary, "MAXIMA-1: a measurement of the cosmic microwave background anisotropy on angular scales of $10^{\prime}-5^{\circ}$," The Astrophysical Journal, vol. 545, no. 1, pp. L5-L10, 2000.

[2] J. H. P. Wu, A. Balbi, J. Borrill et al., "Tests for gaussianity of the MAXIMA-1 cosmic microwave background map," Physical Review Letters, vol. 87, Article ID 251303, 2001.

[3] M. G. Santos, A. Balbi, J. Borrill et al., "Estimate of the cosmological bispectrum from the MAXIMA-1 cosmic microwave background map," Physical Review Letters, vol. 88, Article ID 241302, 2002.

[4] P. D. Mauskopf, P. A. Ade, P. de Bernardis et al., "Measurement of a peak in the cosmic microwave background power spectrum from the North American test flight of boomerang," The Astrophysical Journal, vol. 536, no. 2, pp. L59-L62, 2002.
[5] S. Mosi, P. de Bernardis, G. de Troia et al., "The BOOMERanG experiment and the curvature of the universe," Progress in Particle and Nuclear Physics, vol. 48, no. 1, pp. 243-261, 2002.

[6] N. W. Halverson, E. M. Leitch, C. Pryke et al., "Degree angular scale interferometer first results: a measurement of the cosmic microwave background angular power spectrum," The Astrophysical Journal, vol. 568, no. 1, pp. 38-45, 2002.

[7] G. F. Smoot, C. L. Bennett, A. Kogut et al., "Structure in the COBE differential microwave radiometer first-year maps," The Astrophysical Journal, vol. 396, pp. L1-L5, 1992.

[8] A. H. Jaffe, P. A. R. Ade, A. Balbi et al., "Cosmology from MAXIMA-1, BOOMERANG, and COBE DMR cosmic microwave background observations," Physical Review Letters, vol. 86, no. 16, pp. 3475-3479, 2001.

[9] D. N. Spergel, L. Verde, H. V. Peiris et al., "First-year wilkinson microwave anisotropy probe (WMAP) observations: determination of cosmological parameters," The Astrophysical Journal Supplement Series, vol. 148, no. 1, pp. 175-194, 2003.

[10] D. Spergel, R. Bean, and O. Dore, "Three-Year Wilkinson microwave anisotropy probe (WMAP) Observations: implications for cosmology," The Astrophysical Journal Supplement Series, vol. 170, p. 377, 2007.

[11] P. A. R. Ade, N. Aghanim, C. Armitage-Caplan et al., "Planck 2013 results. XVI. Cosmological parameters," In press, http://arxiv.org/abs/1303.5076.

[12] D. P. Bennett, "Experimental limits on the dark matter halo of the galaxy from gravitational microlensing," Physical Review Letters, vol. 74, pp. 2867-2871, 1995.

[13] P. Ullio and M. Kamioknowski, "Velocity distributions and annual-modulation signatures of weakly-interacting massive particles," Journal of High Energy Physics, vol. 2001, no. 03, p. 049, 2001.

[14] R. Arnowitt and P. Nath, "Event rates in dark matter detectors for neutralinos including constraints from $b \rightarrow s \gamma$ decay," Physical Review Letters, vol. 74, no. 23, pp. 4592-4595, 1995.

[15] R. Arnowitt and P. Nath, "Predictions of neutralino dark matter event rates in minimal supergravity unification," Physical Review D, vol. 54, pp. 2374-2384, 1996.

[16] R. Arnowitt and P. Nath, "Annual modulation signature for the direct detection of milky way WIMP's and supergravity models," Physical Review D, vol. 60, no. 4, Article ID 044002, 1999.

[17] A. Bottino, V. de Alfaro, N. Fornengo, A. Morales, J. Puimedon, and S. Scopel, "Exploring the supersymmetric parameter space by direct search for WIMPs," Physics Letters B, vol. 402, no. 1-2, pp. 113-121, 1997.

[18] J. Ellis and R. A. Flores, "Elastic supersymmetric relic-nucleus scattering revisited," Physics Letters B, vol. 263, pp. 259-266, 1991.

[19] J. Ellis and R. A. Flores, "Prospects for neutralino detection with a ${ }^{73} \mathrm{Ge}+{ }^{76} \mathrm{Ge}$ detector," Physics Letters $B$, vol. 300, no. 1-2, pp. 175-182, 1993.

[20] J. Ellis and R. A. Flores, "Implications of LEP on laboratory searches for dark matter neutralinos," Nuclear Physics B, vol. 400, pp. 25-36, 1993.

[21] J. Ellis and L. Roszkowski, "Supergravity dark matter," Physics Letters B, vol. 283, pp. 252-260, 1992.

[22] M. E. Gómez, G. Lazarides, and C. Pallis, "Supersymmetric cold dark matter with Yukawa unification," Physical Review D, vol. 61, no. 12, Article ID 123512, 2000. 
[23] M. E. Gómez and J. D. Vergados, "Cold dark matter detection in SUSY models at large $\tan \beta$," Physics Letters $B$, vol. 512, no. 3-4, pp. 252-260, 2001.

[24] S. B. Gudnason, C. Kouvaris, and F. Sannino, "Dark matter from new technicolor theories," Physical Review D, vol. 74, Article ID 095008, 2006.

[25] S. Nussinov, "Some estimates of interaction in matter of neutral technibaryons made of colored constituents," Physics Letters B, vol. 279, no. 1-2, pp. 111-116, 1992.

[26] R. Foot, "Mirror \& hidden sector dark matter in the light of new CoGeNT data," Physics Letters B, vol. 703, no. 1, pp. 7-13, 2011.

[27] R. Foot, H. Lew, and R. R. Volkas, "A model with fundamental improper spacetime symmetries," Physics Letters B, vol. 272, no. 1-2, pp. 67-70, 1991.

[28] V. K. Oikonomou, J. D. Vergados, and C. C. Moustakidis, "Direct detection of dark matter rates for various wimps," Nuclear Physics B, vol. 773, no. 1-2, pp. 19-42, 2007.

[29] G. Servant and T. M. P. Tait, "Is the lightest Kaluza-Klein particle a viable dark matter candidate?" Nuclear Physics B, vol. 650, no. 1-2, pp. 391-419, 2003.

[30] J. D. Vergados, "On the direct detection of dark matter," in The Invisible Universe: Dark Matter and Dark Energy, vol. 720 of Lecture Notes in Physics, pp. 69-100, Springer, Berlin, Germany, 2007.

[31] J. Collar and F. T. Avignone III, "Diurnal modulation effects in cold dark matter experiments," Physics Letters B, vol. 275, no. 1-2, pp. 181-185, 1992.

[32] A. K. Drukier, K. Freese, and D. N. Spergel, "Detecting cold dark-matter candidates," Physical Review D, vol. 33, no. 12, pp. 3495-3508, 1986.

[33] N. W. Evans, C. M. Carollo, and P. T. de Zeeuw, "Triaxial haloes and particle dark matter detection," Monthly Notices of the Royal Astronomical Society, vol. 318, no. 4, pp. 1131-1143, 2000.

[34] J. D. Vergados, "Modulation effect for supersymmetric dark matter detection with asymmetric velocity dispersion," Physical Review D, vol. 62, no. 2, Article ID 023519, 2000.

[35] C. S. Cochanek, "The mass of the milky way," The Astrophysical Journal, vol. 457, p. 228, 1996.

[36] J. Vergados, S. N. Hansen, and O. Host, "Impact of going beyond the Maxwell distribution in direct dark matter detection rates," Physical Review D, vol. 77, Article ID 023509, 2008.

[37] G. Gelmini and P. Gondolo, "Adiabatic and isocurvature perturbations from inflation: power spectra and consistency relations," Physical Review D, vol. 64, no. 12, Article ID 123504, 2001.

[38] A. M. Green, "Potential weakly interacting massive particle signature for the caustic ring halo model," Physical Review D, vol. 63, no. 10, Article ID 103003, 2001.

[39] P. Sikivie, "Caustic rings of dark matter," Physics Letters B, vol. 432, no. 1-2, pp. 139-144, 1998.

[40] P. Sikivie, "Caustic ring singularity", Physical Review D, vol. 60, no. 6, Article ID 063501, 1999.

[41] J. D. Vergados, "Searching for supersymmetric dark matter: the directional rate and the modulation effect due to caustic rings," Physical Review D, vol. 63, no. 6, Article ID 063511.

[42] C. Copi, J. Heo, and L. Krauss, "Directional sensitivity, WIMP detection, and the galactic halo," Physics Letters B, vol. 461, no. 1-2, pp. 43-48, 1999.

[43] A. M. Green, "Effect of halo modeling on weakly interacting massive particle exclusion limits," Physical Review D, vol. 66, no. 8, Article ID 083003, 2002.
[44] A. S. Eddington, "The distribution of stars in globular clusters," Monthly Notices of the Royal Astronomical Society, vol. 76, pp. 572-585, 1916.

[45] D. Merritt, "Spherical stellar systems with spheroidal velocity distributions," The Astronomical Journal, vol. 90, pp. 1027-1037, 1985.

[46] D. Owen and J. D. Vergados, "New velocity distribution for cold dark matter in the context of the Eddington theory," The Astrophysical Journal, vol. 589, no. 1, pp. 17-28, 2003.

[47] J. Vergados and D. Owen, "Direct dark matter event rates with a velocity distribution in the Eddington approach," Physical Review D, vol. 75, no. 4, Article ID 043503, 2007.

[48] R. Wojtak, E. L. Łokas, G. A. Mamon, S. Gottlöber, A. Klypin, and Y. Hoffman, "The distribution function of dark matter in massive haloes," Monthly Notices of the Royal Astronomical Society, vol. 388, no. 2, pp. 815-828, 2008.

[49] M. Fornasa and A. Green, "A self-consistent phase-space distribution function for the anisotropic dark matter halo of the milky way," Physical Review D, vol. 89, no. 6, Article ID 063531, 2013.

[50] P. H. Nguyen and J. F. Pedraza, "Anisotropic models for globular clusters, galactic bulges, and dark halos," Physical Review D, vol. 88, no. 6, Article ID 064020, 2013.

[51] C. A. Agón, J. F. Pedraza, and J. Ramos-Caro, "Kinetic theory of collisionless self-gravitating gases: post-newtonian polytropes," Physical Review D, vol. 83, no. 12, Article ID 123007, 2011.

[52] N. Bozorgnia, R. Catena, and T. Schwetz, "Anisotropic dark matter distribution functions and impact on WIMP direct detection," Journal of Cosmology and Astroparticle Physics, vol. 2013, no. 12, article 050, 2013.

[53] D. Hunter, "Derivation of the anisotropy profile, constraints on the local velocity dispersion, and implications for direct detection," Journal of Cosmology and Astroparticle Physics, vol. 2014, no. 02, article 023, 2014.

[54] H. C. Plummer, "On the problem of distribution in globular star clusters," Monthly Notices of the Royal Astronomical Society, vol. 71, pp. 460-470, 1911.

[55] A. Natarajan, C. Savage, and K. Freese, "Probing dark matter streams with CoGeNT," Physical Review D, vol. 84, no. 10, Article ID 103005, 2011.

[56] M. Kuhlen, M. Lisanti, and D. Speregel, "Direct detection of dark matter debris flows," Physical Review D, vol. 86, no. 6, Article ID 063505, 2002.

[57] J. F. Navarro, C. S. Frenk, and S. D. M. White, "The structure of cold dark matter halos," Astrophysical Journal Letters, vol. 462, no. 2, pp. 563-575, 1996.

[58] J. Binney and S. Tremain, Galactic Dynamics, Princeton University Press, Princeton, NJ, USA, 2008.

[59] D. Merritt, "Distribution functions for spherical galaxies," Monthly Notices of the Royal Astronomical Society, vol. 214, no. 1, pp. 25P-28P, 1985.

[60] L. Osipkov, "Spherical systems of gravitating bodies with an ellipsoidal velocity distribution," Soviet Astronomy Letters, vol. 5, pp. 42-44, 1979.

[61] S. H. Hansen, B. Moore, M. Zemp, and J. Stadel, "A universal velocity distribution of relaxed collisionless structures," Journal of Cosmology and Astroparticle Physics, vol. 2006, no. 01, article 014, 2006.

[62] J. D. Vergados, "Debris flows in direct dark matter searches: the modulation effect," Physical Review D, vol. 85, no. 12, Article ID 123502, 2012. 
[63] A. Green, "Effect of realistic astrophysical inputs on the phase and shape of the weakly interacting massive particles annual modulation signal," Physical Review D, vol. 68, Article ID 023004, 2003.

[64] A. Green, "Erratum in: effect of realistic astrophysical inputs on the phase and shape of the weakly interacting massive particles annual modulation signal, Physical Review D, vol. 68, Article ID 023004, 2003," Physical Review D, vol. 69, Article ID 109902, 2004.

[65] J. D. Vergados and C. C. Moustakidis, "WIMP event rates in directional experiments: the diurnal variation signature," Central European Journal of Physics, vol. 9, no. 3, pp. 628-643, 2011.

[66] C. C. Moustakidis, D. Owen, and J. Vergados, "Realistic asymmetric velocity distributions obtained in Eddington approach and their application to to dark matter searches.," to be published, 2014. 

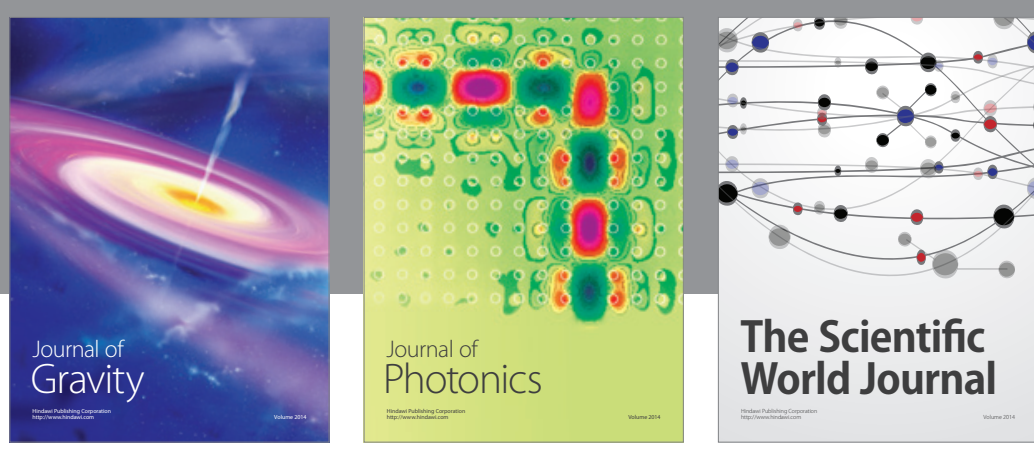

The Scientific World Journal
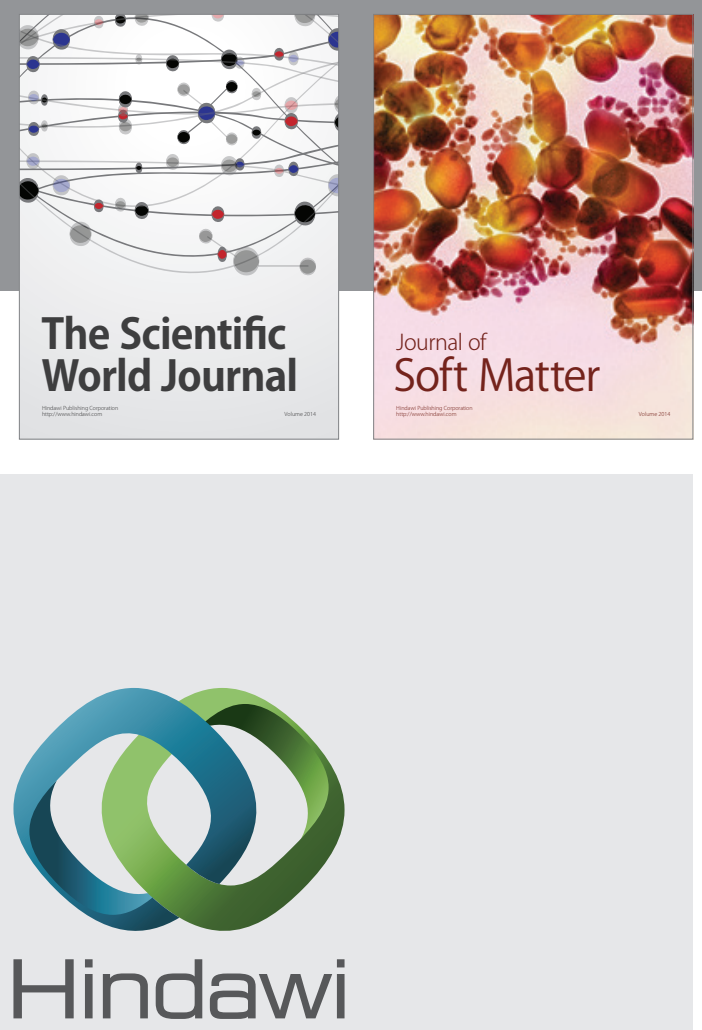

Submit your manuscripts at

http://www.hindawi.com

nternational Journal of

Statistical Mechanics
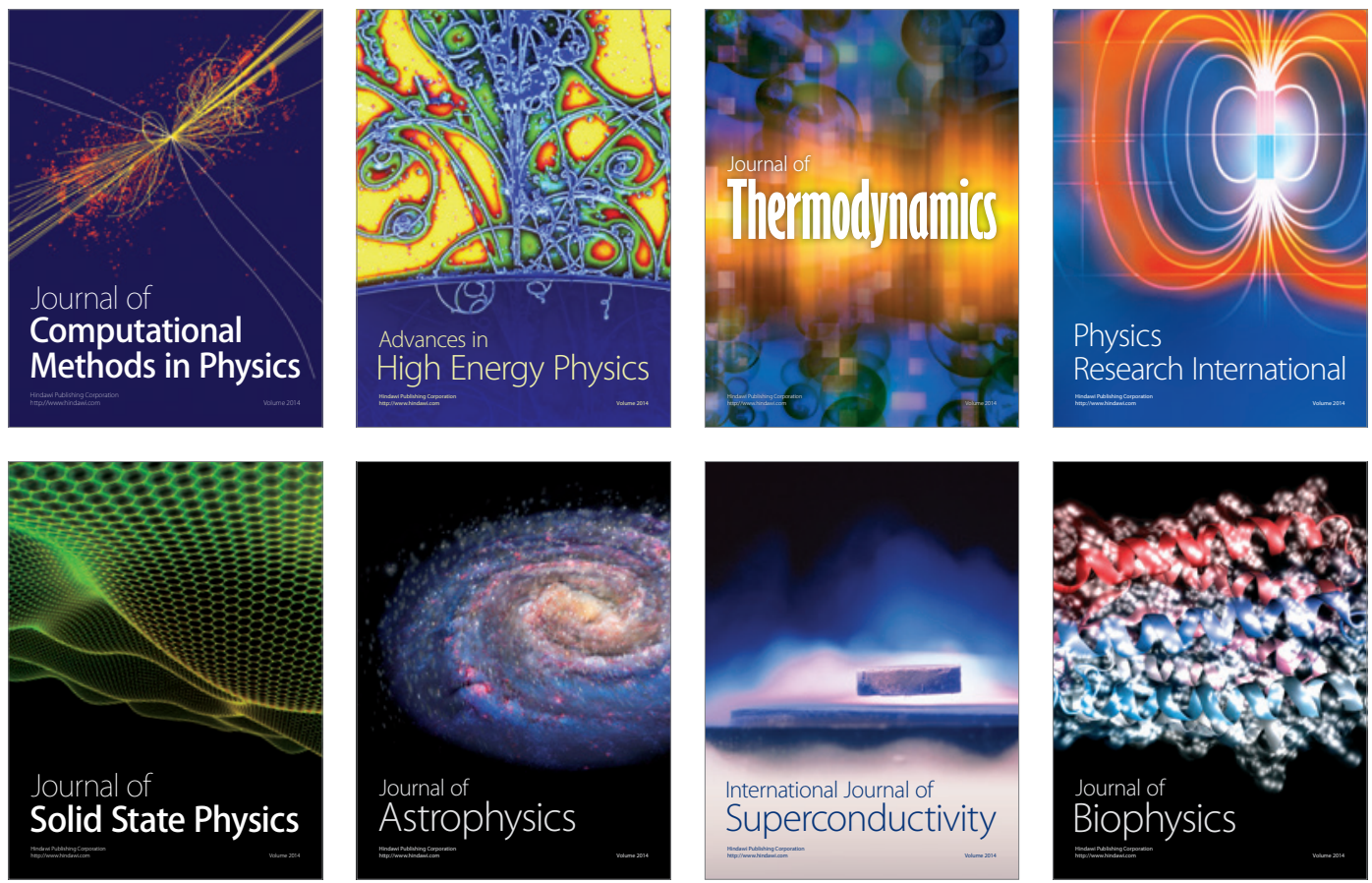
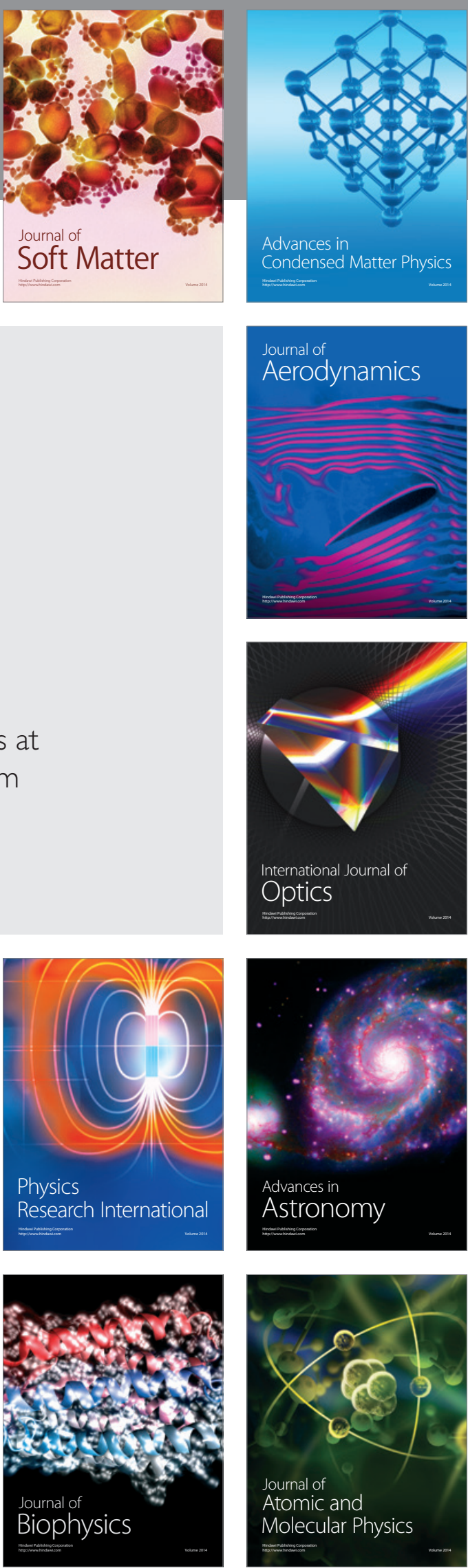